\title{
Investigation and Control of Ultrafast Laser-Induced Nanoscale Patterns in Bulk Fused Silica
}

\author{
R. Stoian, K. Mishchik, G. Cheng, M. Zamfirescu, C. Mauclair, C. d'Amico \\ Laboratoire Hubert Curien, UMR 5516 CNRS, Université de Lyon, Université Jean Monnet, 42000 Saint Etienne, France \\ Author e-mail address: razvan.stoian@univ-st-etienne.fr
}

\begin{abstract}
Refractive index changes in a-SiO ${ }_{2}$ consist of positive index variations or regular nanoscale patterns. We spectroscopically reveal the electronic and structural transformation of glass in the self-organized structures, indicating bond breaking and oxygen deficiency. We equally propose a method of real time control of nanogratings formation under the action of ultrashort laser pulse with variable envelopes. Application as polarizing optical devices is discussed.
\end{abstract}

\section{Introduction}

Local refractive index changes are the building blocks of laser-induced 3D optical functions in transparent materials, e.g. fused silica. Ultrashort pulses could induce either positive or negative isotropic smooth refractive index changes (usually denoted as type I) or produce regular nanoscale self-arranged layered structures resulting in form birefringence (type II nanogratings - Fig. 1) [1-3]. Particularly the latter phenomenon is an intriguing physical manifestation that allows the development of embedded polarization functions. A spectroscopy study reveals the particular electronic and structural transformation of glassy matter in the self-organized subwavelength structures, indicating bond breaking and the abundance in oxygen deficiency. As the spontaneous arrangement is intermediated by electronic excitation, we equally propose a method of real time control of nanogratings formation under the action of laser pulse with programmable variable envelopes. Relying on the advantage of intrinsic anisotropies, the application potential in terms of polarization sensitive optical devices is discussed.

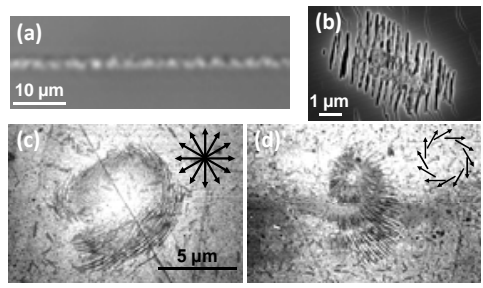

Fig. 1 Type II laser-generated traces and the underlying nanogratings in a- $\mathrm{SiO}_{2}$. (a) Phase contrast microscopy image of type II modification traces. (b) Cross-section of the traces showing the nanoscale arrangement. (c,d) Bulk nanograting patterns induced by radial and azimuthal polarization.

\section{Discussion}

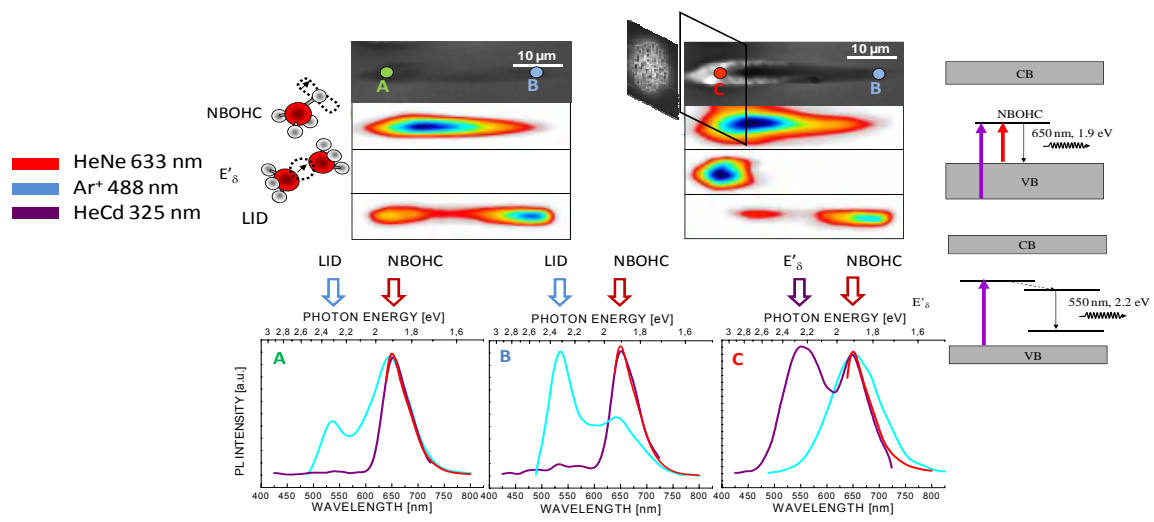

Fig. 2 Particular electronic transformations in a- $\mathrm{SiO}_{2}$, revealed by photoluminescence spectroscopy in a space resolved manner. Type I (left) and type II (right) regimes are depicted.

A relevant question refers to the structural modifications characterizing the glass network in type I and type II regimes. Photoluminescence (PL) studies performed in a space-resolved manner indicate the presence of defects in 
the irradiated regions. HeNe $633 \mathrm{~nm}$ resonant excitation of NBOHC centers probed by 1.9 eV PL reveals a preferential generation of NBOHC in the positive index regions via a probable precursor self-trapping mechanism. Type II modifications and especially the nanopatterned regions show a different type of defect spatial distribution. $\mathrm{Ar}^{+}$excitation at $488 \mathrm{~nm}$, though relaxed via NBOHC PL at $1.9 \mathrm{eV}$ does not resonantly excite these levels, an internal conversion process being responsible for a transfer between a primary excited defect and NBOHC. These defects, also visible in blue luminescence excited by $\mathrm{Cd} 325 \mathrm{~nm}$ sources can be related to oxygen deficiency centers and $\mathrm{E}_{\delta}{ }_{\delta}$ defects. This indicates a dose-specific nature and spatial repartition of defects (Fig. 2). Complementary $\mu$ Raman investigations confirm a densification scenario for positive index changes. Nevertheless, energy balance considerations question if a phase transition related to a significant increase of temperature can be achieved in type I traces, putting forward a concept of "cold" defect-assisted densification. Thermomechanical effects related to pressure waves, compaction and rarefaction, with signs of low viscosity regions take place in type II traces [4].

The dose dependence of the structural modification indicates that control via electronic excitation may be possible for the nanostructured patterns. Using an open loop based temporal shaping procedure with feedback derived from UV diffraction on nanogratings, tunability of the nanogratings period can be achieved, with the smallest period for pulses around 1 ps [3]. Nonlinear pulse propagation calculations indicate a maximum electronic excitation density in these conditions.

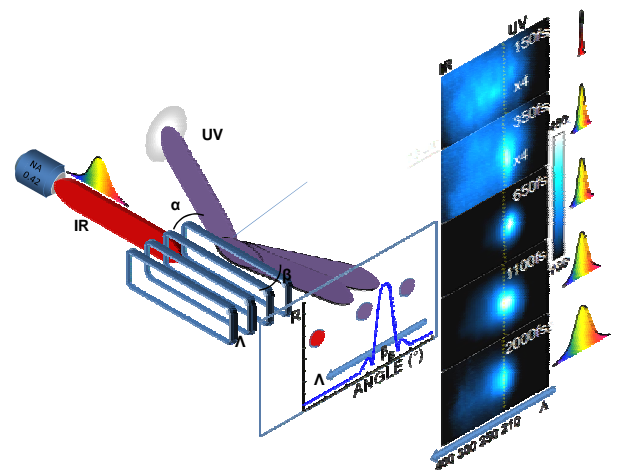

Fig. 3 Tunable ultrafast laser-induced nanogratings periods as a function of the pulse temporal shape visualized via UV diffraction on periodic patterns.

(a)
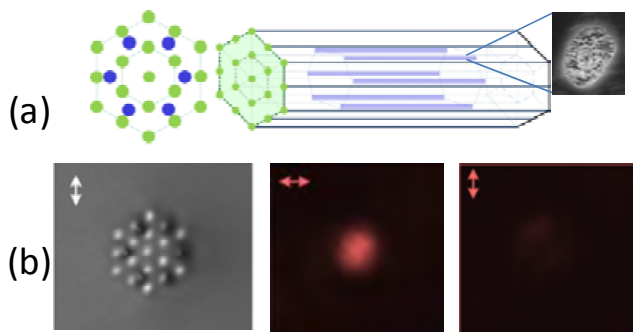

Fig. 4 Polarization large mode area guiding concepts (a) and demonstration (b) using a multicore design.

In terms of optical functionalities, the capability of low-loss guiding of type I traces can be combined with the anisotropic nature of type II nanogratings. The E-field dependent scattering of these subwavelength periodic patterns leads to additional polarization functions. A large mode area guiding trace combining type I multicore traces in hexagonal arrangements and type II scattering defects is presented in Fig. 4.

In conclusion, we indicated structural and electronic transformations related to type I and type II photoinscription domains, demonstrated control options via envelope time design and suggested polarization sensitivity.

\section{References}

[1] G. Cheng, K. Mishchik, C. Mauclair, E. Audouard, and R. Stoian, "Ultrafast laser photoinscription of polarization sensitive devices in bulk silica glass," Opt. Express 17, 9515-9525 (2009).

[2] K. Mishchik, G. Cheng, G. Huo, I. M. Burakov, C. Mauclair, A. Mermillod-Blondin, A. Rosenfeld, Y. Ouerdane, A. Boukenter, O. Parriaux, and R. Stoian, "Nanosize structural modifications with polarization functions in ultrafast laser irradiated bulk fused silica," Opt. Express 18, 24809-24824 (2010)

[3] C. Mauclair, M. Zamfirescu, G. Cheng, J.P. Colombier, E. Audouard, and R. Stoian, "Control of ultrafast laser-induced bulk nanogratings in fused silica via pulse time envelopes" Opt. Express 20, 12997-13005 (2012).

[4] K. Mishchik, C. d'Amico, P. K. Velpula, C. Mauclair, Y Ouerdane, A. Boukenter, and R. Stoian, "Ultrafast laser-induced electronic and structural modifications in bulk fused silica" Opt. Mat. Express 3, 67 (2013). 